\title{
Biomaterials used in stem cell therapy for spinal cord injury
}

\begin{abstract}
Spinal cord injury (SCI) is a common, severe damage to the central nervous system. Here, we discuss the use of biomaterials for stem cell transplantation in preclinical and clinical studies for the treatment of patients with SCI, because cell culture materials could influence the differentiation fate of stem cells, and not act only as carriers or scaffolds for delivery of stem cells and their differentiated cells. Therefore, the effects of cell culture materials on stem cell differentiation fate have been discussed. A direct injection of stem cells is the easiest method to transplant stem cells into the site of SCI. However, the stem cell solution tends to leak out from the injection site. Biomaterials such as fibrin have been used to reduce scarring at the transplantation site and facilitate the integration of transplanted stem cells or progenitor cells in animal models of SCI. Transplantation of stem cells using biomaterials (scaffolds or hydrogels) has been reported to be effective for the treatment of SCI in animal models. It would be necessary to investigate the optimal chemical structure, porosity, and morphology of biomaterials used for the transplantation of stem cells.
\end{abstract}

Keyword: Biomaterial; Stem cell; Hydrogel; Stem cell therapy; Spinal cord injury; Clinical trial 